\title{
Antiretroviral Therapy During Pregnancy in HIV Infected Women
}

\author{
Srinivasan $\mathrm{K}^{1^{*}}$, Kiruthiga $\mathrm{G}^{2}$, Manickavalli. $\mathrm{E}^{3}$, Angelin. $\mathrm{P}^{4}$ \\ ${ }^{1,2,3}$ Associate Professor, Department of Pharmaceutical Chemistry, Nandha College of \\ Pharmacy, Erode, Tamilnadu \\ ${ }^{4}$ Assistant Professor, Department of Community Health Nursing, Nandha College of \\ Nursing, Erode, Tamilnadu. \\ Email:sriudha@gmail.com¹, kiruthiga@nandhapharmacy.org², \\ manickavallielangovan@gmail.com³ ${ }^{3}$ Angelpaulraj10594@gmail.com ${ }^{4}$ \\ ${ }^{*}$ Corresponding Author
}

\begin{abstract}
A focal portion of HIV administration in pregnancy is the use of Sculpture. Knowledge from the NSHPC is used in this blended methodology to explore the relation between maternal identities. The following key pointers are birth and home length. Some include not obtaining ART during breastfeeding, evident maternal HIV viral conveyance burden, and vertical HIV transmission. To discuss women's experiences of taking or not taking ART during pregnancy. A research on the treatment of ART in maternity times of women is made by the dialogical production between quantitative and subjective discoveries.
\end{abstract}

Keywords: Pregnancy, NSHPC, ART

\section{Introduction}

The use of antiretroviral therapy is a focal feature of HIV administration in pregnancy (ART). HIV is an illness that causes AIDS (Human Immunodeficiency Virus) (Acquired Immunodeficiency Syndrome). As they can daily, an AIDS tainted person can not fend off diseases and is more vulnerable with diseases, some tumors and other medical problems that can be life-threatening In breastfeeding, the clinical symptoms for ART are usually direct. During breastfeeding, all women determined to have HIV are advised to take ART to avoid vertical spread of the infection. For their own wellbeing, a few women may have been on medication for quite a while and will hence remain throughout their pregnancy on a similar drug scheme. Others will initiate medication for their own well-being during breastfeeding, either on the grounds that they are at risk of a serious illness or that their CD4 tally has slipped below the criteria for initial treatment, just as for vertical transmission counteraction. A third women's meeting will not need care for their own well-being, and the sole symbol for ART will be vertical expectation. Despite the conditions, most women are advised to undergo care from 14 to 24 weeks of pregnancy at the latest. Therefore, there are major contrasts within this gathering of ladies taking ART, as seen by the history of women taking ART and the clinical motives concealed from their care. We may begin to recognise an unpredictability from now on that misrepresents the simple straightforwardness of clinical laws. In addition, there are major divisions that are at the forefront of HIV care during breastfeeding. During breastfeeding, women living with HIV are urged to take medications to preserve their child's strength, the same moment when other women are advised to exercise alertness when ingesting some product, whether diet or medication. Take-up and commitment to ART is the basis for HIV therapy during pregnancy to reduce the infectious load of maternal HIV in the blood and vagina, thus reducing the risk of vertical transmission. In addition, ART is the way to recover invulnerable ability and preserve long-term well-being if a woman has a compromised resistant structure. Ectopic births and pregnancies leading to an end or unnatural birth period were forbidden by the review of the taking-up of Material, since it would be far-fetched if it was a symbol or freedom to take ART. Possible perplexing considerations. Includes maternal age, history of opioid use infusion, locale declaration, HIV detecting timing and gestational age. In women who had more than one birth, vigorous norm errors were used to describe possible groupings of maternal-level results.

\section{Parental HIV pathological load at delivery}

Mother-to-kid transmission is the stage at which the virus is transmitted to her baby by an HIV-positive woman. This can happen during labour, during work and 
commuting, or during breastfeeding. Without care, during pregnancy and transmission, approximately 1530 percent of children intended for HIV-positive mothers will become infected with HIV. By breast feeding, a further 5-20 percent will get tainted. The key vertical propagation mechanism is the absence of maternal biological suppression at delivery and may be attributed to any sub-optimal reaction (as a result of pharmacokinetics of ART during pregnancy, interactions with other medication or maternal drug resistance) Or bad drug adherence. After sufficient readiness, commence ART at the earliest opportunity to steer and continue with ART during pregnancy, conveyance, and from that point on long-lasting. The ART should be begun quickly regardless of whether eligible pregnant women are late in pregnancy (counting the persons that are present after 36 weeks of incubation). This ART can only be begun at ART focuses, henceforth all efforts need to be made to ensure that pregnant women hit ART focuses. Viral load study at conception was limited to live singleton deliveries of women who were administered HAART during pregnancy. Owing to the risk of maternal co-infections, such as syphilis, which may raise the viral load of HIV, stillbirths have been omitted. Pregnancies in women undergoing mono/dual antiretroviral treatment were omitted because virological inhibition would not be likely to benefit from these regimens. A regimen of three or more antiretroviral drugs has been described as HAART. The nearest recorded maternal HIV viral load to delivery, estimated between twenty-eight days before and seven days after delivery, was described as viral load at delivery. The viral load for transmission was graded as undetectable or detectable;' undetectable.' Analyses considered as confounders were maternal age, history of injection drug use, first viral load in pregnancy, first CD4 count in pregnancy and HAART regimen. The relation between observable viral load at delivery and younger maternal age at delivery, higher earliest antenatal viral load, lower earliest antenatal CD4 count, HAART regimen and shorter ART length was found in the univariate study.

\section{Vertical transmission of HIV}

In HIV testing, the patient is regularly allowed the use of ART in labour, effective conveyance administration and shirking of breastfeeding. There is discussion about the link between vertical transmission and maternal identity. Vertical propagation research was confined to singleton live births. For this examination, no woman reported more than one HIV-tainted child blunders that were not needed. Based on comprehensive polymerase chain response (PCR) or HIV counteracting agent data, baby disease status was assigned to uninfected or polluted.

The ART regimen can continue to be provided to pregnant women who are already undergoing an NVPbased ART regimen. If pregnancy is recognized before 28 days of gestation, EFV should be stopped and replaced with NVP (No Lead in Dose Required) or if a woman is diagnosed as pregnant after 28 days of gestation, EFV should be stopped and replaced with NVP (No Lead in Dose Required) . There is no evidence of abortion/termination of pregnancy in the first trimester of pregnancy in women exposed to EFV. From that stage on, the CD4 cell count reduces during pregnancy due to pregnancy-related haemodilution, like clockwork according to guidelines. In this way, a decrease in overall CD4 may not really show immunological decrease in a pregnant lady embracing ART in comparison with CD4 estimates preceding pregnancy and should be deciphered with alarm. Maternal era, history of infusing substance usage, duration of Painting, CD4 scan at conveyance, gestational age at conveyance, form of conveyance and baby sex were possible confounders considered in the following study. Despite being a perceived risk factor for vertical dissemination as it was presumably going to be on the causal route, viral conveyance load was omitted from the examination. Adherence was the fundamental interfering aspect between all variables of transparency and vertical transmission, and the relation between adherence and vertical transmission would conflict with the viral strain of transmission. Mothers with HIV are recommended not to breastfeed whenever it is appropriate, practical, affordable, sustainable and healthy to use breast milk supplementary formula. Breast pathologies are baby risk of HIV infected mothers during breastfeeding (cracked nipples, mastitis, or engorgement). Poor maternal diet, mouth sores or an inflamed $\mathrm{Gl}$ tract in infants, mixed feeding involves early HIV disease in the mother: breast milk along with other diets, extended breast feeding (6-18 months).

\section{Conclusion}

Although all women took ART during breastfeeding, this introduced different difficulties for others. Not many specifics impaired their adherence; but I learned from conversations with specialists in medical services that different virological concealment had not been done by them, indicating powerless adherence. Sickness, retching, nausea and abdominal torment were endured by several ladies. These actual signals were a wellspring of exceptional nervousness, with the need for excellent obedience to restrict the danger of vertical transmission intensely taken into account by multiple ladies. During pregnancy, most women take Craft, with $80 \%$ achieving virological concealment by conveyance and not many instances. In the age of a positive maternal and feminine character, women focus on taking ART during breastfeeding, using it as a significant 
apparatus. In addition, the condition of ART inside a transnational healthcare environment where biomedicine is well-known and readily accessible. In any event, taking ART includes battling for some women, involving antiretroviral practice to address questions about consequences, harmfulness, and viability. The use of ART was promoted to a limited degree by the intense desire of women to take ART to achieve socially approved jobs as moms and broad partnerships defined by "care" with HIV antenatal expert groups. Suggestions can be more expanded, streamlined and combined to speed up the accelerated worldwide scaling up, ensuring neutral entry.

\section{References}

1. Dadhwal V, Sharma A, Khoiwal K, Deka D, Sarkar P, Vanamail P. Pregnancy outcomes in HIV-infected women: experience from a tertiary care center in india. Int. J. MCH AIDS 6(1), 75 (2017)

2. Salters K, Loutfy $M$, de Pokomandy A et al. Pregnancy incidence and intention after HIV diagnosis among women living with HIV in Canada. PLoS ONE 12(7), e0180524 (2017).

3. Bagkeris E, Malyuta R, Volokha A et al. Pregnancy outcomes in HIV-positive women in Ukraine, 2000-12 (European Collaborative Study in EuroCoord): an observational cohort study. Lancet HIV 2(9), e385-e392 (2015).

4. Tookey PA, Thorne C, van Wyk J, Norton M. Maternal and foetal outcomes among 4118 women with HIV infection treated with lopinavir. Ritonavir during pregnancy: analysis of population-based surveillance data from the national study of HIV in pregnancy and childhood in the United Kingdom and Ireland. BMC Infect. Dis. 16(1), 65 (2015).

5. Baroncelli S, Tamburrini E, Ravizza M et al. Pregnancy outcomes in women with advanced HIV infection in Italy. AIDS Patient Care STDS 25(11), 639-645 (2011).

6. Moodley D, Esterhuizen TM, Pather T, Chetty V, Ngaleka L. High HIV incidence during pregnancy: compelling reason for repeat HIV testing. AIDS 23(10), 1255-1259 (2009)

7. van Eijk AM, De Cock KM, Ayisi JG et al. Pregnancy interval and delivery outcome among HIV-seropositive and HIV-seronegative women in Kisumu, Kenya. Trop. Med. Int. Health 9(1), 15-24 (2004).

8. Sh B, Eshagi M, Farhoodi B, Gooya M, Kamali K, Tayeri K. HIV prevention from mother to child: transmission protocols for the Islamic Republic of Iran; Ministry of Health. Center Dis. Control 5, 1 (2008). 